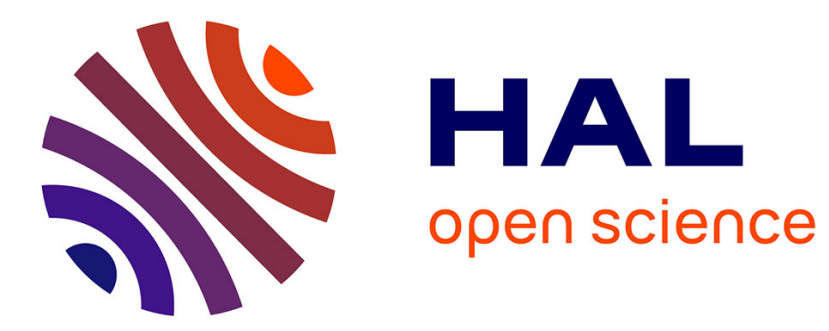

\title{
Copy Sensitive Graphical Code Quality Improvement Using a Super-Resolution Technique
}

Iuliia Tkachenko, Florentin Kucharczak, Christophe Destruel, Olivier Strauss, William Puech

\section{- To cite this version:}

Iuliia Tkachenko, Florentin Kucharczak, Christophe Destruel, Olivier Strauss, William Puech. Copy Sensitive Graphical Code Quality Improvement Using a Super-Resolution Technique. ICIP: International Conference on Image Processing, Oct 2018, Athens, Greece. 10.1109/ICIP.2018.8451839 . hal-01909478

\section{HAL Id: hal-01909478 \\ https://hal.science/hal-01909478}

Submitted on 31 Oct 2018

HAL is a multi-disciplinary open access archive for the deposit and dissemination of scientific research documents, whether they are published or not. The documents may come from teaching and research institutions in France or abroad, or from public or private research centers.
L'archive ouverte pluridisciplinaire HAL, est destinée au dépôt et à la diffusion de documents scientifiques de niveau recherche, publiés ou non, émanant des établissements d'enseignement et de recherche français ou étrangers, des laboratoires publics ou privés. 


\section{COPY SENSITIVE GRAPHICAL CODE QUALITY IMPROVEMENT USING A SUPER-RESOLUTION TECHNIQUE}

\author{
I. Tkachenko ${ }^{1}$ \\ ${ }^{1}$ Laboratory Hubert Curien, UMR CNRS 5516 \\ University Lyon, UJM-Saint-Etienne \\ 18 Rue Professeur Benoît Lauras \\ 42000 Saint-Etienne, France
}

\author{
F. Kucharczak ${ }^{2}$, C. Destruel ${ }^{2}$, O. Strauss ${ }^{2}$, W. Puech ${ }^{2}$ \\ ${ }^{2}$ Laboratory LIRMM, UMR CNRS 5506 \\ University of Montpellier \\ 161, rue Ada \\ 34090 Montpellier, France
}

\begin{abstract}
The authentication of printed documents is an important problem these days. Numerous authentication techniques have been proposed in the relevant literature. One of the most promising solutions uses copy-sensitive graphical codes made of particular patterns. The two Level QR (2LQR) code uses specific textured patterns in order to ensure the sensitivity to duplication process. The authentication test of this code is based on comparing the correlation values between the original and the printed-and-scanned codes with a pre-determined threshold. The weakest feature of this technique is the rejection of authentic codes due to the small gap between correlation values for authentic and duplicated code. In this paper, we propose to reduce the number of false-negative results of the authentication test by using a very competitive SuperResolution (SR) technique. The experimental results show the significant improvement of correlation values when using the images printed-and-scanned once, without increasing the correlation values of duplicated codes. Therefore, the $2 \mathrm{LQR}$ code copy sensitivity is not affected by the suggested quality improvement process.
\end{abstract}

Index Terms - printed document authentication, printand-scan process, super-resolution, image quality improvement

\section{INTRODUCTION}

The increasing use of numerical documents does not decrease the popularity and usefulness of printed documents. Unfortunately, the number of counterfeited printed documents increases every year due to cheap, accessible and high quality of printer and scanner devices. To counteract this fraud, different authentication systems were created to protect the document against falsification: watermarking techniques [1], self-embedding techniques [2] and use of copy sensitive graphical codes [3].

Using copy sensitive graphical codes is a very promising and cheap technique to secure valuable printed documents.
This technique relies on the loss of information due to the stochastic nature of Print-and-Scan (P\&S) process [4]: after each $P \& S$ process, the structure of the patterns forming the graphical code changes. This change allows to distinguish original documents from their copies. This kind of code can be represented by a maximum entropy image, generated using a secret key [5], or by textured patterns [6].

In this paper, we focus on the two Level QR (2LQR) code proposed in [6] which is an enhanced 2D barcode with a copy sensitive ability. This $2 \mathrm{LQR}$ code has two storage levels. The first level is public, accessible for everybody who uses a standard QR code reader. The second level is based on replacing the black modules of the conventional $\mathrm{QR}$ code by $q$ of those textured patterns $P_{l}, l=1, \ldots, q$ that are sensitive to $\mathrm{P} \& \mathrm{~S}$ process. This level is used to store a supplementary message and also to authenticate the $2 \mathrm{LQR}$ code. Due to the sensitivity of the patterns to the $\mathrm{P} \& S$ process, a copy of the proposed 2LQR code can be successfully distinguished from its original print [7]. Nevertheless, the gap between original and copied $2 \mathrm{LQR}$ codes is not big and the control general conditions (impact of scanner, light) could be very different from one case to another: thus several authentic codes can be rejected by the authentication test used.

Here we consider increasing the quality of captured version of a code by using a multi-frame Super-Resolution (SR) approach. This allows us to improve the textured pattern recognition and to increase the gap between original and scanned codes. To solve the aforementioned problem, we envisage to use a novel and robust multi-frame SR method presented in [8]. We study its effectiveness for image quality improvements after $\mathrm{P} \& \mathrm{~S}$ process. As the aforementioned SR method used is non-regularized, we decided to also compare the results with the regularized version (regularized selection framework) of this algorithm proposed in [9]. It is the only consistent explicit regularization method for [8]. Both regularized and non-regularized algorithms are proved to be very competitive compared to their state-of-the-art multi-frame SR competitors.

The rest of the paper is organized as follows. The printed 
document authentication system based on graphical codes is presented in Section 2. The image quality improvement process using SR is discussed in Section 3. In Section 4 we describe the performed experiments and evaluate the obtained results. Finally, the conclusions and future paths are considered in Section 5.

\section{AUTHENTICATION SYSTEM BASED ON COPY SENSITIVE GRAPHICAL CODES}

The legitimate life-cycle of a document protected by a 2LQR code consists of the following parts 1) valuable document generation, where the authentic document and $2 \mathrm{LQR}$ code are put together and printed using a particular printer, 2) document authentication, where the document is scanned using a specific device and then, after textured pattern detection, the authentication test is performed. If the authentication test has a positive result, the scanned document is considered as authentic. The authentication process of $2 \mathrm{LQR}$ code is based on correlation values [10] and a pre-calculated authentication threshold.

In the adversary path, the printed document is scanned, reconstructed and re-printed by attacker. Then, this document is submitted to the authentication process: scan, pattern detection and authentication test. The forged document has undergone through two P\&S processes. Thus, due to the loss of information due to the stochastic nature of each $\mathrm{P} \& \mathrm{~S}$ process, the structure of the textured patterns has been significantly changed, and therefore the document is rejected as non-authentic. This authentication methodology is presented in Fig. 1.

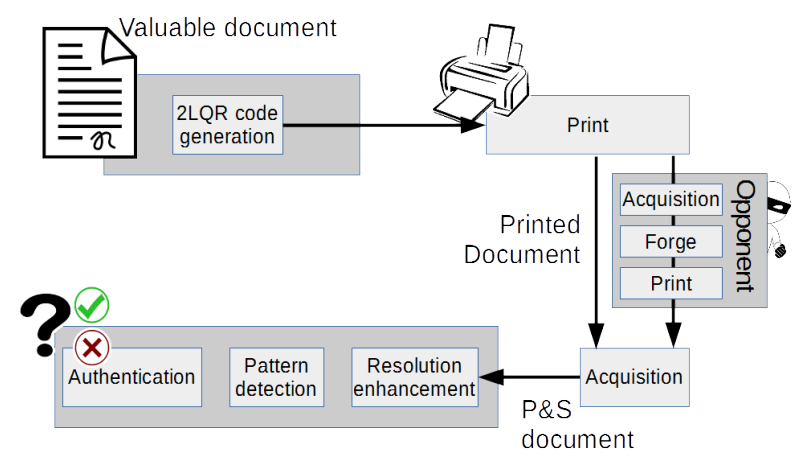

Fig. 1. Considered authentication system using graphical codes.

The 2LQR code generation and authentication processes are proposed in [6]. An authentication threshold $T h$ is determined during the pre-trial phase. The authentication test consists of comparing the mean value of $\operatorname{cor}_{i}, i=1, \ldots, n$ (where $\operatorname{cor}_{i}$ is the maximal correlation value between the $\mathrm{P} \& \mathrm{~S}$ pattern and original patterns and $n$ is the number of patterns in $2 \mathrm{LQR}$ code) with threshold $T h$. The document is said to be authentic if this mean value is bigger than $T h$ (mean $\left.\left(\operatorname{cor}_{1}, \ldots, \operatorname{cor}_{n}\right) \geq T h\right)$. The studied duplication and naive attacks [7, 10] show the problems of authentication process. The correlation values of several authentic codes are quite small and thus leads sometimes to its rejection. Therefore, we consider a pre-processing step that increases the image resolution via an SR technique in this paper. This kind of reconstruction is likely to compensate for the degradation due to the stochastic nature of the $P \& S$ process.

\section{QUALITY IMPROVEMENT PROCESS}

The main goal of this paper is to focus on a quality enhancement process that improves the image quality after scanning in order to improve the efficiency of the authentication test. Such an authentication test has to differentiate originals from copies: we want to improve the gap between both classes, after P\&S process. This work is the first to deal with copysensitive codes.

We use an SR technique for image quality improvement. The main idea of this proposition is to improve the resolution of the captured $2 \mathrm{LQR}$ code image. The enhanced image is then used as any captured image to determine whether the code is authentic or not. The authentication test with quality improvement pre-processing is presented in Algorithm. 1.

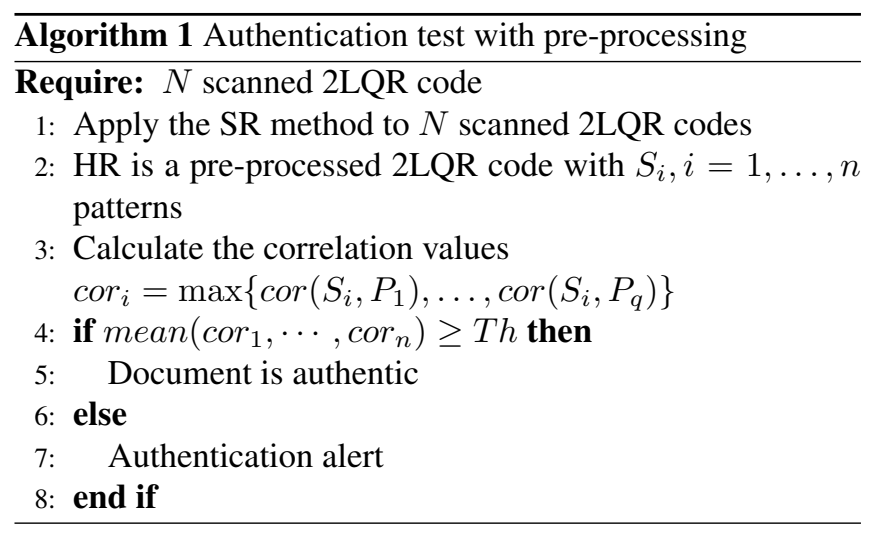

Each printed $2 \mathrm{LQR}$ code is scanned $N$ times. Each scan is considered as a Low Resolution (LR) image. A gray level High Resolution (HR) image is then reconstructed based on this set of $N$ scanned LR images. Then, this HR image passes through the authentication test based on correlation value comparison. In Section 4, we show that the correlation values of an HR image are higher in comparison with that of the image obtained directly after P\&S process.

Our goal is to produce a more reliable authentication test that increases the gap of correlation scores between original and counterfeited codes. To do that we apply the pre-processing step by improving the image quality with SR technics before the authentication test. 


\section{EXPERIMENTS}

In this section, we present the database used and the SR method setup. Then we describe the experiments and analyze the obtained results.

\subsection{Database description}

The database with original and $\mathrm{P} \& \mathrm{~S} 2 \mathrm{LQR}$ codes was created in order to study the effects of SR methods on P\&S images. In total we have 20 sets of samples with different 2LQR codes. Each set contains 15 different prints and scans of the same code. The $2 \mathrm{LQR}$ codes used are of size $49 \times 49$ patters that corresponds to a version 8 of $\mathrm{QR}$ code. The supplementary message is encoded using three different textured patterns. Each 2LQR code has been printed (with a Brother HL-4150CDN printer) and scanned (with a Canon LIDE210 scanner) with a 600 dpi resolution. An example of $2 \mathrm{LQR}$ code as well as the textured patterns used are illustrated in Fig. 2.

Note that the 600dpi resolution for the capture step is chosen to be close to realistic use cases: even if higher resolutions are now available, various devices used can not provide more than 600dpi.

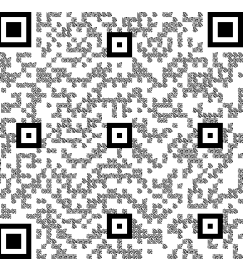

(a)

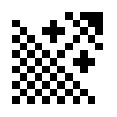

(b)

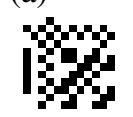

(c)

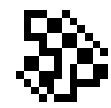

(d)
Fig. 2. Examples of $2 \mathrm{LQR}$ code and textured patterns used: a) 2LQR code, b) Pattern 1, c) Pattern 2, d) Pattern 3.

\subsection{SR method setup}

We propose to improve the image quality of the copy sensitive code by using a new, competitive and robust method based on LR multi-frame acquisition [8] and its regularized version [9]. The idea underlying this test is to compensate for the stochastic variability of the scanning process by reconstructing an HR image based on a set of different LR images. For the testing, we used subsets of 5,10 and 15 LR images for each sample. Both reconstruction and regularization processes are iterative. In our tests, we performed 15 iterations for reconstruction and 8 for regularization. One particularity of the proposed non-regularized SR method, is that the reconstructed image is interval-valued. Thus, as in [8], we use the central image of each reconstructed interval-valued image.

\subsection{Image quality improvement of scanned codes}

As a first result, in all experiments, the resolution quality of the $2 \mathrm{LQR}$ code image was improved by SR reconstruction (see Fig. 3). Each HR image was constructed using $5-15$ P\&S 2LQR code images. Fig. 3 presents an example of the increased resolution of a $P \& S$ LQR code by using a nonregularized SR technique - see on Fig. 3.a an original LR image, its HR non-regularized reconstruction using 10 and 15 LR images on Fig. 3.b - Fig. 3.c, and its HR regularized reconstruction using $10 \mathrm{LR}$ images on Fig. 3.d.

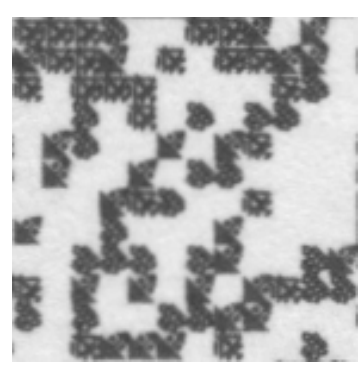

(a)

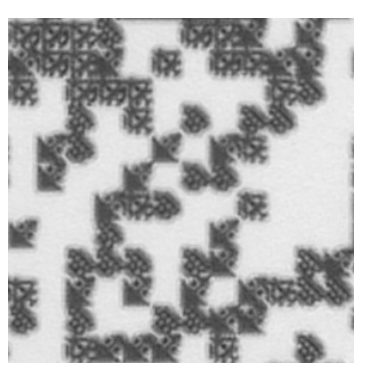

(c)

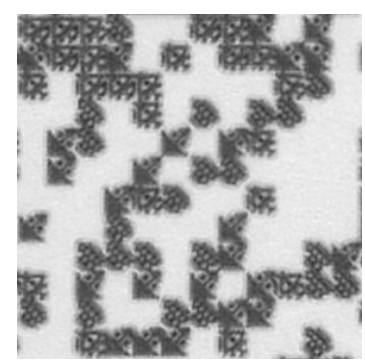

(b)

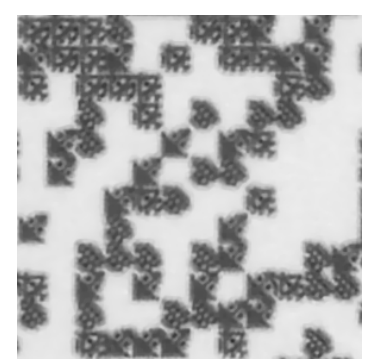

(d)
Fig. 3. (a) A zoomed part of a P\&S $2 L Q R$ code, (b) Its HR version using non-regularized method with 10 images, (c) Its HR version using non-regularized method with 15 images and (d) Its HR version using regularized method with 10 images.

Our goal is to increase the correlation values of patterns after $\mathrm{P} \& S$ process. Table 1 presents the comparison of mean correlation scores with and without an SR enhancement. This SR enhancement obviously increases the mean correlation values (at least up to 0.35 for whole 2LQR code image). This means that the number of authentic code rejections during authentication test decreases. As noted by [11], the SR enhancement increases with the number of images but reaches a limit that is inherent to the reconstruction process. This also can be observed with the correlation results: using more than 10 images does not lead to sensible improvement.

Table 1 shows the changes of correlation values depending on number of the LR subsample size used for HR image estimation. We can observe that the correlation results are worse for the regularized reconstruction method than for the nonregularized reconstruction method for all subsets. The regularization method allows to obtain better images in terms of 


\begin{tabular}{|l|c|c|c||c|}
\hline & Pattern 1 & Pattern 2 & Pattern 3 & 2LQR \\
\hline \hline \multicolumn{5}{|c|}{ After P\&S } \\
\hline & 0.2900 & 0.2647 & 0.3706 & 0.3084 \\
\hline \hline \multicolumn{5}{|c|}{ After SR non-regularized } \\
\hline 5 images & 0.3697 & 0.3074 & 0.4611 & 0.3794 \\
\hline 10 images & 0.3724 & $\mathbf{0 . 3 0 7 6}$ & 0.4634 & $\mathbf{0 . 3 8 1 1}$ \\
\hline 15 images & $\mathbf{0 . 3 7 3 5}$ & 0.3053 & $\mathbf{0 . 4 6 3 5}$ & 0.3808 \\
\hline \multicolumn{5}{|c|}{ After SR regularized } \\
\hline 5 images & 0.3560 & 0.2983 & 0.4155 & 0.3566 \\
\hline 10 images & 0.3587 & 0.2984 & 0.4447 & 0.3673 \\
\hline 15 images & 0.3575 & 0.2947 & 0.4420 & 0.3648 \\
\hline
\end{tabular}

Table 1. Mean correlation values for each type of patterns and for whole $2 \mathrm{LQR}$ code before and after SR reconstruction.

PSNR, but not necessarily improve the correlation. For the non-regularized SR method, as explained earlier, using subsets of 10 LR images seems to be sufficient for our framework.

\subsection{SR impact on counterfeited codes}

As discussed in Section 3, it is necessary to evaluate the correlation levels for counterfeited codes. Our goal is to increase the difference between correlation scores of original and counterfeited codes. In order to study this difference, we construct a database of $2 \mathrm{LQR}$ code printed and scanned twice using 1200 dpi resolution. Then, the image quality of these codes was improved using the same setup described earlier for SR reconstruction. Finally, the authentication test with pre-determined authentication threshold $T h=0.3$ was performed under these $\mathrm{HR}$ double $\mathrm{P} \& \mathrm{~S} 2 \mathrm{LQR}$ code images. The comparison of correlation measures is illustrated in Fig. 4. We note that the use of SR methods for original P\&S images increases the correlation values. Nevertheless, the correlation values are almost stable for counterfeited (printed and scanned twice) images using the same SR method.

We have to make clear that the SR technique is used as a preprocessing step in order to increase the quality of codes during authentication test. The aim of this section is not to propose any attack technique: the possibilities are too large and it is not possible to guess which can be used by an attacker. One can imaging several attacks using any SR technique between two P\&S operations. However, the HR image needs to be binarized before second $\mathrm{P} \& \mathrm{~S}$ operation as common printers deal only with black and white information. The choice of good binarization strategy is an open question, that is why we do not perform the attacks using the SR technique in this paper.

Nevertheless, the results illustrated in Fig. 4 show that the use of the SR methods from [9] cannot improve the correlation values in duplicated code, thus the duplication codes cannot pass the authentication test. Therefore, the main property of

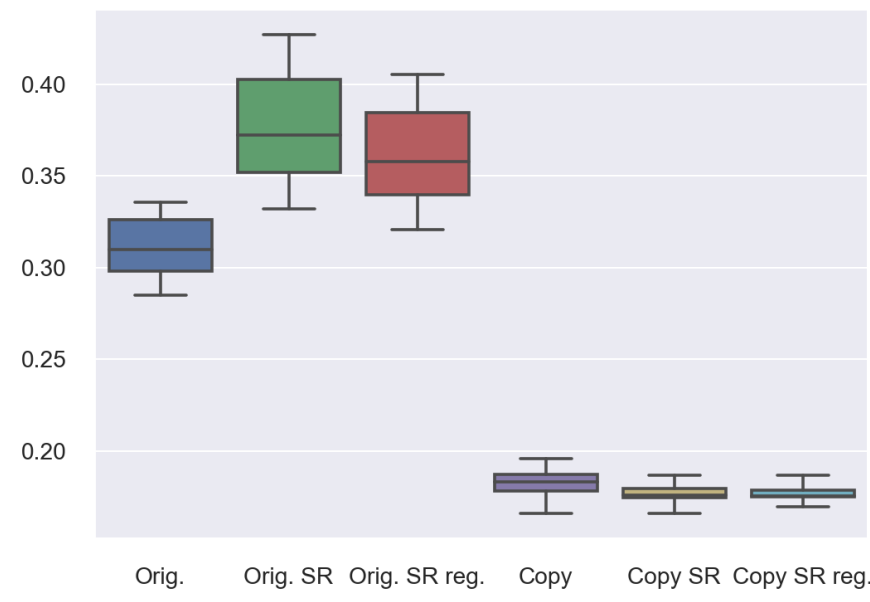

Fig. 4. Authentication test results for codes printed-andscanned twice without and with SR pre-processing.

2LQR code - sensitivity to duplication process - is not affected by the image quality improvement using these SR methods.

\section{CONCLUSION}

In this paper we propose to improve an authentication system based on copy sensitive graphical codes by using a SuperResolution (SR) method. Never until now have SR techniques been used for this type of application. The authentication we consider is based on detecting whether the $2 \mathrm{LQR}$ code, that is used to authenticate the document, has been printed once or printed then scanned then printed. This detection is based on a correlation measure between the original graphical patterns that are used to construct the $2 \mathrm{LQR}$ code and its printed-then-scanned version. The experiments we report here show that the resolution enhancement induced by the SR process increases the correlation values between original and printed-and-scanned patterns when the document is printedthen-scanned only once, but not if the document has been printed-then-scanned more than once. Therefore, by considering the SR as a preprocessing, the robustness of the authentication is increased: there are less false negatives. In the future, we would like to study the $2 \mathrm{LQR}$ code estimation attacks based on SR methods, and also to propose a SR regularization method to directly integrate the binarization in the reconstruction process (for example adding the information on the number of expected black pixels in each patterns). As second path, we would like to perform tests with singleimage SR methods to reconstruct the known patterns. Finally, we need to perform the complete attack channel with a bigger database of different copy-sensitive graphical codes and several printers/scanners. 


\section{REFERENCES}

[1] J. Fridrich, "Visual hash for oblivious watermarking," in Electronic Imaging. International Society for Optics and Photonics, 2000, pp. 286-294.

[2] R. Villán, S. Voloshynovskiy, O. Koval, F. Deguillaume, and T. Pun, "Tamper-proofing of electronic and printed text documents via robust hashing and data-hiding," in Electronic Imaging 2007. International Society for Optics and Photonics, 2007, pp. 65 051T-65 051T.

[3] J. Picard, "Digital authentication with copy-detection patterns," in Electronic Imaging 2004. International Society for Optics and Photonics, 2004, pp. 176-183.

[4] C. Baras and F. Cayre, "2D bar-codes for authentication: A security approach," in Signal Processing Conference (EUSIPCO), Proceedings of the 20th European, 2012, pp. 1760-1766.

[5] J. Picard and J. Zhao, "Techniques for detecting, analyzing, and using visible authentication patterns," May 3 2011, uS Patent 7,937,588.

[6] I. Tkachenko, W. Puech, C. Destruel, O. Strauss, J.M. Gaudin, and C. Guichard, "Two-level QR code for private message sharing and document authentication," IEEE Transactions on Information Forensics and Security, vol. 11, no. 3, pp. 571-583, 2016.

[7] I. Tkachenko, W. Puech, O. Strauss, C. Destruel, and J.M. Gaudin, "Printed document authentication using two level QR code," in 2016 IEEE International Conference on Acoustics, Speech and Signal Processing (ICASSP). IEEE, 2016, pp. 2149-2153.

[8] F. Graba, F. Comby, and O. Strauss, "Non-additive imprecise image super-resolution in a semi-blind context," IEEE Trans. Image Processing, vol. 26, no. 3, 2017.

[9] F. Kucharczak, C. Mory, O. Strauss, F. Comby, and D. Mariano-Goulart, "Regularized selection: a new paradigm for inverse based regularized image reconstruction techniques," in 2017 IEEE International Conference on Image Processing (ICIP). IEEE, 2017.

[10] I. Tkachenko, C. Destruel, O. Strauss, and W. Puech, "Sensitivity of different correlation measures to printand-scan process," Electronic Imaging, vol. 2017, 2017.

[11] P. Milanfar, Super-resolution imaging. CRC Press, 2010 . 